Rev. Biol. Trop., 47(1-2): 123-132, 1999

www.ucr.ac.cr www.ots.ac.cr www.ots.duke.edu

\title{
Cangrejos braquiuros (Brachyura) del arrecife de Cabo Pulmo-Los Frailes y alrededores, Golfo de California, México
}

\author{
María del Socorro García-Madrigal y José Rolando Bastida-Zavala \\ Consultoría e Investigación Ambiental, A.C. (CINAM), Apdo. Postal 466, Chetumal, QROO, 77000, México. Fax: \\ (983) 204-47; e-mail: ms_garcia_mhotmail.com \\ El Colegio de la Frontera Sur (ECOSUR), Apdo. Postal 424, Chetumal 77000 QROO \\ Recibido 21-I-1998. Corregido 11-XI-1998. Aceptado 3-XII-1998
}

\begin{abstract}
Fragments of the coral Pocillopora elegans collected in 1988 and 1989 produced 281 specimens of brachyurans. There were 30 species and subspecies in eight families, and 22 are new records for the Cabo Pulmo-Los Frailes reef. Including earlier records, there are now 61 species and subspecies (19 families) from the area. Majidae had the largest number of species (23), followed by Panopeidae (9), and Xanthidae (6). Most abundant were Trapezia ferruginea (71 specimens), Theleophrys cristulipes (39) and Microcassiope xantusii xantusii (29). The species were sorted out in four zoogeographic groups: Indopacific (8.5\%), Amphiamerican (5.1\%), Eastern Pacific (76.3\%), and Mexican Pacific endemics (10.1\%). The reef represents only $0.004 \%$ of the Gulf area but harbors $21 \%$ of all species recorded from the gulf of California, western coast of Baja California Sur and exclusive economic zone of Nayarit and Sinaloa.
\end{abstract}

Key words: Coral reef, faunistic, Pocillopora, zoogeography, checklist.

En los arrecifes coralinos del Pacífico oriental, principalmente en los de Panamá, Costa Rica y de las islas Galápagos, Malpelo y Gorgona, se han realizado, desde 1970, estudios sistemáticos sobre la estructura de las comunidades, ecología, depredación y fisiología (ver ref. en Cortés y Murillo 1985 y Glynn 1991). En el Pacífico americano no había arrecifes coralinos durante el Pleistoceno, los actuales son muy jóvenes, pues comenzaron a desarrollarse hace 4500 a 5600 años (Cortés 1986), además son pequeños y con una distribución discontinua debido a la reducida superficie de la plataforma continental, la existencia de amplias zonas costeras con sustratos arenosos, la presencia de varios ríos permanentes, el elevado aporte anual de precipitación pluvial, las surgencias y la incidencia de huracanes y tormentas tropicales (Reyes-Bonilla 1993).
Por su parte, los arrecifes del Pacífico mexicano han sido poco estudiados a pesar de que el primer registro de un coral hermatípico fue hace más de 150 años; sin embargo, en la última década se han intensificado los trabajos sobre las formaciones coralinas del golfo de $\mathrm{Ca}$ lifornia y del sur de México, siendo el arrecife de Cabo Pulmo-Los Frailes el más conocido, seguido por los arrecifes de isla Socorro y las formaciones coralinas de Guerrero y Oaxaca (Reyes-Bonilla 1993).

$\mathrm{El}$ arrecife de Cabo Pulmo-Los Frailes se localiza en la parte sur de la península de Baja California (entre los $23^{\circ} 24^{\prime}-23^{\circ} 27^{\prime} \mathrm{N}$ y $109^{\circ} 24^{\prime}-109^{\circ} 26^{\prime} \mathrm{W}$ ), y a pesar de sus pequeñas dimensiones es de los más grandes del Pacífico oriental, con 150 ha de cobertura coralina (Reyes-Bonilla 1993). Esta área tan reducida contiene, probablemente, la mayor riqueza específica de invertebrados y peces de todo el 
golfo de California (Reyes-Bonilla 1990, Robinson y Thomson 1992, Bastida-Zavala 1994). El arrecife está compuesto por tres barras paralelas a la costa, de $4 \mathrm{~km}$, de donde se han registrado diez especies de corales hermatípicos, siendo Pocillopora elegans Dana la especie dominante en las barras arrecifales, aunque a mayor profundidad comienza a dominar Porites panamensis Verrill (Reyes-Bonilla 1993). El estatus taxonómico de P. elegans, al menos para el golfo de California, es confuso y está en espera la confirmación de su sinonímia con $P$. verruco$s a$ (Ellis \& Solander) (Reyes-Bonilla 1992).

En los últimos veinte años se han realizado en el arrecife de Cabo Pulmo-Los Frailes diversos trabajos sobre algas, invertebrados y peces (Brusca y Thomson 1977), corales (Reyes-Bonilla 1990), poliquetos (Bastida-Zavala 1991, 1994), además de estudios ecológicos (Robinson y Thomson 1992). Actualmente el arrecife se encuentra protegido bajo la categoría "Parque Nacional" (Anónimo 1998).

Por otra parte, la información sobre braquiuros en el arrecife de Cabo Pulmo-Los Frailes es pobre comparándola con la que existe para el golfo de California; había siete trabajos previos que incluyen 39 especies de braquiuros: Glassell (1936) registró tres especies a $2.5 \mathrm{~km}$ de Cabo Pulmo; Steinbeck y Ricketts (1941) informaron de seis especies más; Garth (1958) registró 16 especies de májidos y dos años después, él mismo (1960), citó seis braquiuros más para Los Frailes; Garth y Stephenson (1966) registraron dos especies de portúnidos en las cercanías del arrecife; Brusca y Thompson (1977) informaron de cinco nuevos registros; y Rodríguez de la Cruz (1987) citó dos especies más para la zona.

El presente trabajo incluye los resultados del análisis de las especies de braquiuros del arrecife de Cabo Pulmo-Los Frailes y alrededores, tanto los registros previos como los obtenidos por nuevos muestreos, y de su composición zoogeográfica.

\section{MATERIALES Y MÉTODOS}

Se realizó una revisión bibliográfica sobre los registros previos de braquiuros del arrecife de Cabo Pulmo-Los Frailes y alrededores, y para complementar la información del área se efectuaron siete campañas de muestreo durante mayo y septiembre de 1988 y 1989. Los sitios de muestreo se ubican en las tres barras coralinas del arrecife de Cabo Pulmo y en el Coral Los Frailes (Cuadro 1, Fig. 1).

El muestreo se llevó a cabo con buceo autónomo, extrayendo fragmentos de colonias, en su mayor parte muertas, del coral hermatípico $P$. elegans, las cuales fueron colocadas in situ dentro de cubetas de plástico con el fin de evitar la pérdida de material; por cada sitio se extrajo una cubeta. Las muestras, dentro de sus respectivas cubetas, fueron sometidas a un choque osmótico cambiando el agua del medio por agua dulce, con el fin de evitar la depredación entre los organismos y/o su autotomía, e inmediatamente fueron fijadas con una solución de formaldehído al $10 \%$. No se tomó nota del volumen de cada muestra de coral, aunque se procuró extraer colonias con dimensiones semejantes. Cada cubeta utilizada tenía 151 de capacidad.

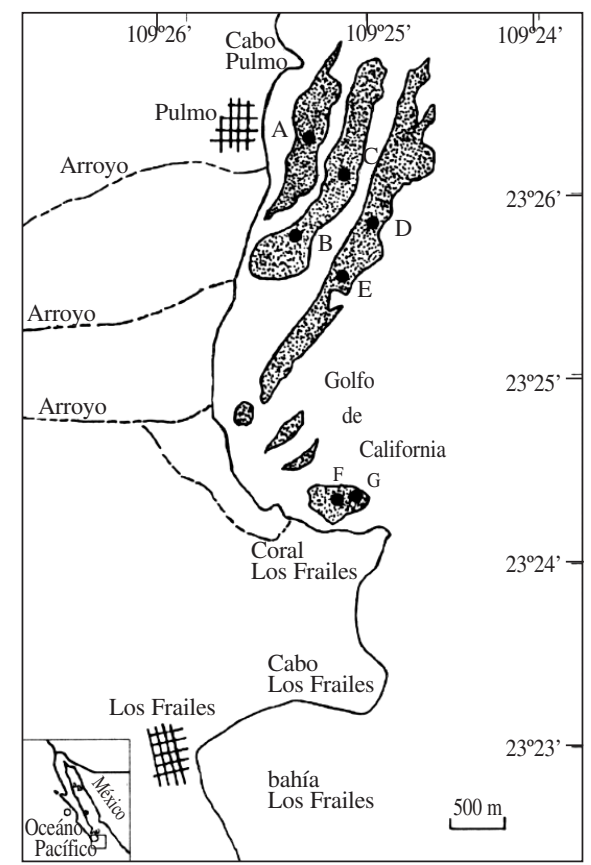

Fig. 1. Area de estudio. A) Primera barra (CP-588), B) segunda barra (CP-589-2), C) segunda barra (CP-989-2), D) tercera barra (CP-589-1), E) tercera barra (CP-989-1), F) Coral Los Frailes (CP-988), y G) Coral Los Frailes (CP-989-3). 


\section{CUADRO 1}

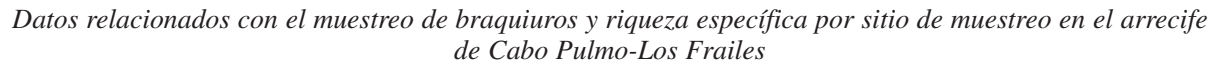

Estación

Sitio

CP-588

CP-589-2

CP-989-2

CP-589-1

CP-989-1

CP-988

CP-989-3

Primera barra
Segunda barra
Segunda barra
Tercera barra
Tercera barra
Coral Los Frailes
Coral Los Frailes

Profundidad

(m)

5

7

4

12

12
17

4

4
No. de ejemplares

32
34
25
23
49
94
24

\begin{tabular}{|c|c|c|}
\hline \multicolumn{3}{|c|}{ Número de especies } \\
\hline por estación & por sitio & acumuladas \\
\hline 5 & 5 & 5 \\
\hline 14 & 17 & 22 \\
\hline 8 & & \\
\hline 13 & 15 & 26 \\
\hline 8 & & \\
\hline 13 & 15 & 30 \\
\hline 7 & & \\
\hline & & tal 30 \\
\hline
\end{tabular}

En el laboratorio se les extrajo a las muestras la solución de formaldehído y se les mantuvo sumergidas en agua dulce, por 24 horas, para eliminar el preservador. Posteriormente las muestras fueron fragmentadas con un martillo geológico, y con ayuda de pinzas se separaron, por grupos taxonómicos, todos los invertebrados encontrados. Los organismos fueron colocados en frascos y preservados en alcohol al $70 \%$.

La identificación de las especies de braquiuros se realizó con ayuda de literatura especializada (Rathbun 1918, 1930, 1937, Garth 1958, Guinot 1967, 1990, Campos 1993). Para el listado de las especies se siguió la clasificación de la superclase Crustacea propuesta por Bowman y Abele (1982), con modificaciones para la superfamilia Xanthoidea (Guinot 1978). Todos los ejemplares se depositaron y catalogaron en la Colección Enrique Rioja de Invertebrados Acuáticos de Consultoría e Investigación Ambiental (CINAM) en Chetumal, Quintana Roo.

Las distribuciones de los braquiuros se obtuvieron de diversas fuentes: Villalobos-Hiriart et al. (1989), Garth (1991) y Hendrickx (1992). Para el análisis de la composición zoogeográfica de los braquiuros del arrecife de Cabo Pulmo-Los Frailes se siguió, con modificaciones, el esquema propuesto por Garth (1991), que consiste de cuatro grupos zoogeográficos:

Indopacífico: Especies presentes en el océano Indico y en ambas costas del océano Pacífico.

Anfiamericano: Especies presentes en la costa del Pacífico oriental y en el Atlántico occidental.

Pacífico oriental: Especies presentes en aguas tropicales y subtropicales de la costa del Pacífico oriental (de bahía Magdalena a Ecuador y Perú).

Endémico al Pacífico mexicano: Especies registradas exclusivamente en el Pacífico de México.

\section{RESULTADOS}

Las muestras contenían 281 ejemplares de braquiuros (Cuadro 1), clasificables en 30 especies y subespecies de diez familias, de las cuales siete especies ya habían sido citadas para el área de estudio (Cuadro 2). Majidae obtuvo la mayor riqueza específica con diez especies; seguida por Panopeidae con seis especies, Xanthidae con cinco, Trapeziidae con tres, y Leucosiidae, Dairididae, Atelecyclidae, Pilumnidae, Pinnotheridae y Cryptochiridae con una especie cada una. Las especies más abundantes en las muestras fueron el trapécido Trapezia ferruginea (71 ejemplares), el májido Theleophrys cristulipes (39) y el panopeido Microcassiope xantusii xantusii (29).

Por otra parte, el número de especies de braquiuros registrados en la literatura para el área de estudio era de 39 especies y subespecies, repartidas en 16 familias. Con las especies registradas en este trabajo, el número de braquiuros para el arrecife de Cabo Pulmo-Los Frailes y alrededores se incrementó a 61 especies y subespecies de 19 familias; manteniéndose las 
mismas familias dominantes en cuanto a riqueza específica: Majidae con 23 especies, Panopeidae con nueve y Xanthidae con seis (Cuadro 2).

Para el análisis de la composición zoogeográfica sólo se tomaron en cuenta las 59 especies identificadas (Cuadro 2). Sólo ocho especies $(13.6 \%)$ tienen amplia distribución, cinco son indopacíficas $(6.8 \%)$ y tres son anfiameri- canas $(5.1 \%)$. Las especies restringidas al Pacífico oriental tropical-subtropical suman 51 (86.4\%), de las cuales seis (10.2\%) son especies o subespecies endémicas al Pacífico mexicano (Cuadro 2): los májidos Hemus analogus, Stenocionops beebei y Thoe sulcata sulcata, el portúnido Portunus xantusii minimus, el panopeido Gonopanope nitida y el pilúmnido $P i$ lumnus stimpsoni.

\section{CUADRO 2}

Listado de las especies de braquiuros del arrecife de Cabo Pulmo-Los Frailes y alrededores, hábitats de recolecta y composición zoogeográfica.

Familia Especie

\begin{abstract}
Registro
\end{abstract}
Hábitat
Grupos zoogeográficos anfiam. P. oriental endémico

Dromiidae

Hypoconcha californiensis Bouvier 1898

Dynomenidae

Dynomene ursula Stimpson 1860

Calappidae

Cycloes bairdii Stimpson 1860

Leucosiidae

Uhlias ellipticus Stimpson 1871

Majidae

Collodes granosus Stimpson 1860

Eupleurodon trifurcatus Stimpson 1871

Euprognatha bifida Rathbun 1893

Hemus analogus Rathbun 1898

Lissa aurivilliusi Rathbun 1898

Microphrys branchialis Rathbun 1898

M. platysoma (Stimpson 1860)

M. triangulatus (Lockington 1877)

Mithrax (Mithraculus) denticulatus Bell 1835

M. (Mithrax) armatus de Saussure 1853

M. (M.) sinensis Rathbun 1892

M. (M.) tuberculatus Stimpson 1860

Paradasygyius depressus (Bell 1835)

Pitho picteti (de Saussure 1853)

P. sexdentata Bell 1835

Podochela hemphilli (Lockington 1877)

P. veleronis Garth 1958

P. vestita (Stimpson 1871)

Stenocionops beebei Glassell 1936

Stenorhynchus debilis (Smith 1871)

Teleophrys cristulipes Stimpson 1860

Thoe sulcata sulcata Stimpson 1860

Tyche lamellifrons Bell 1835

Parthenopidae

Parthenope triangula (Stimpson 1860)

Solenolambrus arcuatus Stimpson 1871

Dairididae

Daira americana Stimpson 1860

Atelecyclidae

Kraussia americana Garth 1939

Pliosoma parvifrons Stimpson 1860

Portunidae

Portunus tuberculatus Rathbun 1888

P. xantusii minimus Rathbun 1898

(7)

7

4

8

3

3
8

3
8

8

8

8

$2,3,8$

$\mathrm{b} ?$
$\mathrm{r}$
$?$
$\mathrm{c}$
$\mathrm{c}$
$\mathrm{b}$
$\mathrm{c}$
$\mathrm{b}$
$\mathrm{c}$
$\mathrm{b}$
$\mathrm{c}$
$\mathrm{c}$
$\mathrm{c}$
$\mathrm{c}$
$\mathrm{b}$
$\mathrm{c}$
$\mathrm{c}$
$\mathrm{c}$
$\mathrm{c}$
$\mathrm{c}$
$\mathrm{c}$
$\mathrm{b}$
$\mathrm{b}$
$\mathrm{b}$
$\mathrm{b}, \mathrm{c}$
$\mathrm{b}$
$\mathrm{b}$
$\mathrm{b}$
$\mathrm{b}$
$\mathrm{b}$
$\mathrm{b}$
$\mathrm{b}$

X

X

X

X

X

X

X

X

X

X

X

X

X

$\mathrm{X}$

(Continúa) 
CUADRO 2: Continuación)

Familia

$$
\text { Especie }
$$

$$
\text { Registro Hábitat indopac. }
$$

Grupos zoogeográficos

Xanthidae

Cycloxanthops vittatus (Stimpson 1860)

Heteractea lunata (Milne Edwards \& Lucas 1843)

Liomera (Liomera) cinctimana (White 1847)

Paractaea sulcata (Stimpson 1860)

Xanthodius sternberghii Stimpson 1859

X. stimpsoni (Milne Edwards 1879)

Mennipidae

Eriphia squamata Stimpson 1859

Panopeidae

Coralliope cf. armstrongi (Garth 1948)

Edwardsium lobipes (Rathbun 1898)

Garthiope sp.

Gonopanope areolata (Rathbun 1898)

G. nitida (Rathbun 1898)

Medaeus spinulifer (Rathbun 1898)

Microcassiope xantusii xantusii (Stimpson 1871) 8

M. xantusii cf. taboguillensis (Rathbun 1907) 8

Micropanope sp.

Pilumnidae

Pilumnus pygmaeus Boone 1927

P. stimpsoni Miers 1886

Trapeziidae

Domecia hispida Eydoux \& Souleyet 1841

Trapezia digitalis Latreille 1825

T. ferruginea Latreille 1825

Pinnotheridae

Juxtafabia muliniarum (Rathbun 1918)

Grapsidae

Grapsus grapsus (Linnaeus 1758)

Pachygrapsus transversus (Gibbes 1850)

Ocypodidae

Ocypode occidentalis Stimpson 1860

Palicidae

Palicus fragilis (Rathbun 1893)

Cryptochiridae

Hapalocarcinus marsupialis Stimpson 1859

anfiam.

P. oriental endémico

8
8
8
8
6
8

8
4
8
4
8
4
8
8
8

c

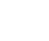

X

$\mathrm{X}$

$\mathrm{X}^{* * *}$

$\mathrm{X}$

$\mathrm{X}$

$\mathrm{X}$

$\mathrm{X}$

$\mathrm{X}$

$\mathrm{X}$

$\mathrm{X}$

$\mathrm{X}$

$\mathrm{X}$

$\mathrm{X}$

X

$\mathrm{X}$

$\begin{array}{lll}8 & \mathrm{c} & \mathrm{X} \\ 2,6,8 & \mathrm{c} & \mathrm{X}\end{array}$

$2,6,8 \quad \mathrm{c}$

$8 \quad \mathrm{c}$

$6 \quad r$

$2 r$

$6 \quad \mathrm{a}$

$1 \quad ?$

8

Total $=61$ especies
$\mathrm{X}$

$\mathrm{X}$

$\mathrm{X}$

$\mathrm{X}$

$\mathrm{X}^{* *}$

$\mathrm{X}$

$\mathrm{X}$

5

3

45

6

* Su ámbito de distribución se extiende hasta California.

** Su ámbito de distribución se extiende hasta Chile.

*** Su ámbito de distribución se extiende hasta California, hacia el norte, y hasta Chile, hacia el sur.

Referencias: 1) Glassell 1936, 2) Steinbeck y Ricketts 1941, 3) Garth 1958, 4) Garth 1960, 5) Garth y Stephenson 1966, 6) Brusca y Thompson 1977, 7) Rodríguez de la Cruz 1987, 8) este trabajo. Hábitat: $c=$ coral, $r=r o c a, b=$ fondo blando sublitoral, a= playa arenosa. Grupo zoogeográfico: indopac.$=$ indopacífico, anfiam. $=$ anfiamericano, P. oriental $=$ Pacífico oriental y endémico $=$ Pacífico mexicano.

\section{DISCUSIÓN}

Faunística y notas ecológicas: Todas las especies encontradas habían sido registradas con anterioridad en otros sitios de la costa mexicana, con la única excepción de Microcassiope xantusii cf. taboguillensis, la cual, de confirmarse su identificación, se consideraría como nuevo registro para las costas de México, extendiendo su ámbito geográfico hacia el norte desde Panamá. Para la identificación completa de Garthiope sp. y Micropanope sp. se requiere recolectar más organismos. En el primer caso el género contiene cuatro especies 
(Guinot 1990, Guinot \& Iliffe 1991) de las cuales ninguna se asemeja a los ejemplares encontrados. En el segundo caso los ejemplares son muy pequeños y están muy deteriorados.

Como las muestras no tienen igual volumen no pueden compararse, salvo con el análisis directo de la riqueza específica: la segunda barra arrecifal presenta la mayor riqueza de braquiuros (17 especies), seguida por la tercera barra (15) y por Coral Los Frailes (15), mientras que la primer barra sólo presentó cinco especies (Cuadro 1, Fig. 1); sin embargo, estas diferencias probablemente son reflejo del esfuerzo de muestreo y/o el volumen de los fragmentos extraídos.

Entre los fragmentos recolectados en todos los sitios, excepto en la segunda barra, venían pequeñas colonias vivas de $P$. elegans, y en ellas se encontraron cuatro especies de braquiuros que habitan principalmente en corales vivos, el criptoquírido Hapalocarcinus marsupialis y los trapécidos Domecia hispida, T. digitalis y $T$. ferruginea. Los últimos tres también son los braquiuros dominantes en los sitios someros, como la primer barra $(75 \%)$ y en el Coral Los Frailes (44\%), mientras que faltan en la segunda barra (4-7 $\mathrm{m}$ de profundidad) y pobremente representados (14\%) en la tercera barra (12-17 m de profundidad). Los trapécidos son comensales obligados que se alimentan del moco excretado por los corales pocilopóridos (Prahl et al. 1978, Castro 1982).

Asimismo, H. marsupialis está presente con siete ejemplares en la primer barra y con uno y dos ejemplares en el Coral Los Frailes y la tercera barra, respectivamente, pero falta en la segunda barra. La explicación a esto es semejante que para los trapécidos, debido a que no se incluyó coral vivo en las muestras de la segunda barra. Para los criptoquíridos es particularmente importante encontrar sitios adecuados en las cimas de las colonias de coral, donde encuentran condiciones favorables en cuanto al movimiento de las corrientes, y a su vez establecen una relación muy estrecha con el coral que los hospeda, debido a que la hembra de estos braquiuros queda prisionera en la cavidad que el coral forma sobre el braquiuro (Garth y Hopkins 1968). La especi- ficidad de los criptoquíridos a sus hospederos es a nivel de familia, y para el caso de los Hapalocarcinus sus hospederos son especies de la familia Pocilloporidae (Kropp 1990).

Comparando la riqueza específica de braquiuros con otras regiones (Cuadro 3) se observa que la riqueza de especies en el arrecife de Cabo Pulmo-Los Frailes y sus alrededores es modesta, en relación con el atolón Enewetak, o las islas Galápagos. Sin duda, la intensidad y tiempo de las campañas de muestreo en estos sitios, así como la superficie contemplada, que por ende implica mayor cantidad de hábitats cubiertos (v.g. Caribe mexicano, área II del Pacífico mexicano), permitieron la obtención de un mayor número de especies. Por ello, creemos que el número de especies de braquiuros en el arrecife de Cabo Pulmo-Los Frailes se incrementaría con estudios más profundos y detallados.

De cualquier forma, en la riqueza específica de los braquiuros de este pequeño arrecife y sus alrededores (menos de $30 \mathrm{~km}^{2}$ ), está representado el $21 \%$ de las 290 especies del área II (sensu Hendrickx 1993) del Pacífico mexicano, región con 704200 km² de piso oceánico que comprende el golfo de California, la costa occidental de Baja California Sur, las áreas costeras de Nayarit y Sinaloa y la zona económica exclusiva (200 millas náuticas) de estos estados (Hendrickx 1993).

Adicionalmente, se tienen registros del hábitat en 55 de los braquiuros del arrecife de Cabo Pulmo-Los Frailes y alrededores (Cuadro 2), dominando el coral (56.9\%), seguido por los fondos blandos sublitorales $(29.3 \%)$, los fondos rocosos $(12.1 \%)$ y la playa arenosa $(1.7 \%)$.

La elevada riqueza de especies puede deberse a la variedad de sustratos que ofrece el arrecife tanto para los braquiuros como para otros invertebrados. Robinson y Thomson (1992) realizaron un análisis de la cobertura de tres sustratos principales en las barras arrecifales, donde el coral vivo representa en promedio el $73.8 \%$, las cabezas de coral muerto cubiertas por algas el $23.66 \%$ y las áreas rocosas conforman el $2.49 \%$.

En el arrecife de Cabo Pulmo-Los Frailes y sus alrededores, el coral vivo es preferido 
principalmente por xántidos, trapécidos, criptoquíridos, entre otros braquiuros; en las cabezas de coral muerto cubiertas por algas se encuentran májidos y xántidos; en las áreas rocosas se encuentran májidos, xántidos y grápsidos (Cuadro 2). En otros hábitats cercanos o asociados al arrecife, como las playas arenosas, se encuentran ocipódidos, y en los fondos blandos sublitorales anexos a las barras arrecifales se obtuvieron drómidos, májidos y partenópidos (Cuadro 2).

Análisis zoogeográfico: Este análisis indica que la fauna de braquiuros del arrecife es principalmente tropical. Comparando estos resultados con trabajos similares (Garth 1991, 1992, García-Madrigal 1994), se observa que los grupos de amplia distribución (indopacífica y anfiamericana), tomados en conjunto (Cuadro 3), son similares entre las islas Galápagos (14.2\%), la bahía de Maruata $(14.6 \%)$ y Cabo Pulmo-Los Frailes (13.6\%); aunque en las islas Revillagigedo estos componentes están mejor representados (26.2\%). Empero, comparándolos por separado se observa que el grupo indopacífico es escaso en bahía Maruata, similar entre las islas Galápagos y Cabo Pulmo-Los Frailes, y más elevada en las islas Revillagigedo; y el componente anfiamericano está mejor representado en bahía Maruata e islas Revillagigedo que en las islas Galápagos y Cabo PulmoLos Frailes (Cuadro 3).

Por su parte, el componente del Pacífico oriental está mejor representado en la bahía Maruata y Cabo Pulmo-Los Frailes, con valores

\section{CUADRO 3}

Comparación entre diferentes trabajos de las composición zoogeográfica y la riqueza específica de los braquiuros del arrecife de Cabo Pulmo-Los Frailes y alrededores.

\begin{tabular}{|c|c|c|c|c|c|c|}
\hline \multirow[b]{2}{*}{ Referencia } & \multirow[b]{2}{*}{ Sitio } & \multicolumn{4}{|c|}{ Grupos zoogeográficos (porcentajes) } & \multirow{2}{*}{$\begin{array}{l}\text { Especies } \\
\text { incluidas }\end{array}$} \\
\hline & & Indopac. & Anfiam. & P. oriental & Endémico & \\
\hline Garth (1991) & Islas Galápagos & 7.5 & 6.7 & 62.5 & $23.3 \mathrm{a}$ & 120 \\
\hline Garth (1992) & Islas Revillagigedo & 14.8 & 11.5 & 63.9 & $9.8 \mathrm{a}$ & 61 \\
\hline García-Madrigal (1994) & Bahía Maruata & 2.4 & 12.2 & 78.1 & $7.3 b$ & 41 \\
\hline Este trabajo & Cabo Pulmo-Los Frailes & 8.5 & 5.1 & 76.3 & $10.1 b$ & 59 \\
\hline
\end{tabular}

Comparación de la riqueza específica entre varios sitios. El arreglo es de mayor a menor superficie cubierta

Referencia

Hendrickx (1993)

Markham et al. (1990)

Garth (1978)

Abele (1976)

Garth (1991)

Garth et al. (1987)

Garth (1992)

García-Madrigal (1994)

Este trabajo
Sitio

Área II del Pacífico de México (ver texto)

Caribe mexicano (Quintana Roo)

Bahamas (Atlántico)

Panamá

Islas Galápagos

Atolón Enewetak e islas Marshall (Pacífico central)

Isla Socorro

Isla Clarión

Isla Clipperton

Bahía Maruata (Michoacán)

Cabo Pulmo-Los Frailes y sus alrededores
Especies incluidas

$$
\begin{gathered}
290^{\mathrm{a}} \\
116 \\
53^{\mathrm{b}} \\
20^{\mathrm{c}} \\
120 \\
291^{\mathrm{d}} \\
36 \\
45 \\
34 \\
41^{\mathrm{e}} \\
61
\end{gathered}
$$

a Todas las especies de braquiuros registradas para esa zona.

$\mathrm{b}$ Material recolectado en las zonas litorales y sublitorales.

c Sólo las especies de braquiuros encontrados en Pocillopora damicornis en el océano Pacífico.

$\mathrm{d}$ Incluye braquiuros terrestres y fósiles.

$\mathrm{e}$ Incluye braquiuros terrestres.

Grupo zoogeográfico: indopac $=$ indopacífico, anfiam. $=$ anfiamericano, P. oriental $=$ Pacífico oriental, endémico $=$ Pacífico mexicano. 
semejantes, más que en las islas Galápagos y Revillagigedo (Cuadro 3). El porcentaje de endemismos (Cuadro 3) es muy elevado en las islas Galápagos (23.3\%), mientras que el resto de los valores son menores, pero similares, en las islas Revillagigedo (9.8\%), bahía Maruata (7.3\%) y Cabo Pulmo-Los Frailes (10.1\%). Es oportuno aclarar que en los dos primeros sitios sólo se refieren al endemismo insular de las islas Galápagos y Revillagigedo, mientras que en la bahía Maruata y Cabo Pulmo-Los Frailes se refiere a las especies endémicas del Pacífico mexicano.

El arrecife de Cabo Pulmo-Los Frailes, además de contener una elevada riqueza de especies de invertebrados (corales, poliquetos y braquiuros), provee también refugio a por lo menos 25 especies de peces (Robinson y Thomson 1992). Su importancia intrínseca se conjunta con la fragilidad, debida a sus pequeñas dimensiones, su situación geográfica, que linda en el límite de los arrecifes coralinos, y por las actividades humanas, que incluyen la pesca deportiva, el turismo de baja densidad y el buceo autónomo; por ello es recomendable que se intensifique el estudio integral de la zona.

\section{AGRADECIMIENTOS}

Se agradece a Patricia Hernández-Cortés, James Ketchum, Rodolfo Moreno y Héctor Reyes-Bonilla (UABCS, La Paz), por su apoyo en la obtención de las muestras. Miguel Angel Díaz-Arredondo (Est. Oceanogr., Ensenada), proporcionó una referencia importante del arrecife de Cabo Pulmo-Los Frailes. La revisión del manuscrito por Luis Soto y Andrea Raz-Guzmán (ICMyL, UNAM) y de Sergio Salazar-Vallejo (ECOSUR, Chetumal), así como los comentarios y sugerencias de tres árbitros anónimos, mejoró la calidad del escrito.

\section{RESUMEN}

El análisis de los fragmentos del coral Pocillopora elegans recolectados en siete campañas de muestreo durante 1988 y 1989 resultó en 281 ejemplares de braquiuros, identificándose 30 especies de ocho familias, de las cuales 22 especies son nuevos registros para el área. Había 39 es- pecies de braquiuros registradas; ahora hay 61 especies de braquiuros, repartidas en 16 familias. Majidae contiene el mayor número de especies (23), seguida por Panopeidae (9) y Xanthidae (6). Las especies más abundantes fueron Trapezia ferruginea (71 ejemplares), Theleophrys cristulipes (39) y Microcassiope xantusii xantusii (29). Todas las especies se agruparon en cuatro grupos zoogeográficos: indopacífico (8.5\%), anfiamericano $(5.1 \%)$, Pacífico oriental $(76.3 \%)$ y endémico del Pacífico mexicano (10.1\%). Las especies de braquiuros de este arrecife (con sólo $0.004 \%$ de la superficie de la región) representan el $21 \%$ de todas las especies registradas para la región del golfo de California, costa occidental de Baja California Sur, áreas costeras de Nayarit y Sinaloa y zona económica exclusiva de estos estados.

\section{REFERENCIAS}

Abele, L.G. 1976. Comparative species composition and relative abundance of decapod crustaceans in marine habitats of Panamá. Mar. Biol. 38: 263-278.

Anónimo. 1998. Hoja Web del Instituto Nacional de Ecología, México. Consultado en: http://www. ine.gob.mx/ucanp/parque.html (31 de julio de 1998).

Bastida-Zavala, J.R. 1991. Previous list of polychaetes (Annelida: Polychaeta) from Cabo Pulmo-Los Frailes Reef, B.C.S., Mexico. Bull. Mar. Sci. 48: 584.

Bastida-Zavala, J.R. 1994. Poliquetos (Annelida: Polychaeta) del arrecife coralino de Cabo Pulmo-Los Frailes, B.C.S., México. Contr. Zool. CINAM 2: 1-49.

Bowman, T.E. \& L.G. Abele. 1982. Classification of the Recent Crustacea. p. 1-27 In L.G. Abele (ed.). The Biology of Crustacea: Systematics, the Fossil Record, and Biogeography. Vol. 1, Academic, Nueva York.

Brusca, R.C. \& D.A. Thomson. 1977. Pulmo Reef: The only "coral reef" in the Gulf of California. Cienc. Mar. (1975) 2: 37-53.

Campos, E. 1993. Systematics and taxonomic remarks on Pinnotheres muliniarum Rathbun, 1918 (Crustacea: Brachyura: Pinnotheridae). Proc. Biol. Soc. Wash. 106: 92-101.

Castro, P. 1982. Notes on symbiotic decapod crustaceans from Gorgona Island, Colombia, with a revision of the Eastern Pacific species of Trapezia (Brachyura, Xanthidae), symbionts of scleractinian corals. An. Inst. Inv. Mar. Punta Betín 12: 9-17.

Cortés N., J. 1986. Biogeografía de corales hermatípicos: el Istmo Centro Americano. An. Inst. Cienc. Mar. Limnol., UNAM 13: 297-304. 
Cortés N., J. \& M.M. Murillo. 1985. Comunidades coralinas y arrecifes del Pacífico de Costa Rica. Rev. Biol. Trop. 33: 197-202.

García-Madrigal, M.S. 1994. Brachyura (Crustacea: Decapoda) de la Bahía de Maruata, Michoacán, México. Contr. Zool. CINAM 1: 1-58.

Garth, J.S. 1958. Brachyurans of the Pacific coast of America: Oxyrhyncha. Allan Hancock Pac. Exped. 21: $1-854$.

Garth, J.S. 1960. Distribution and affinities of the brachyuran crustacea. Syst. Zool. 9: 105-122.

Garth, J.S. 1978. Marine biological investigations in the Bahamas. 19. Decapoda Brachyura. Sarsia 63: 317-333.

Garth, J.S. 1991. Taxonomy, distribution, and ecology of Galápagos Brachyura. p. 123-145. In M.J. James (ed.). Galápagos Marine Invertebrates. Plenum, Nueva York.

Garth, J.S. 1992. The brachyuran crabs of the Revillagigedo islands, Colima, Mexico, with remarks on insular endemism in the Eastern tropical Pacific. Proc. San Diego Soc. Nat. Hist. 24: 1-6.

Garth, J.S., J. Haig \& J.W. Knudsen. 1987. Crustacea Decapoda (Brachyura and Anomura) of Enewetak atoll. p. 235-261. In D.M. Devaney, E.E. Reese, B.L. Burch \& P. Helfrich (eds.). The Natural History of Enewetak Atoll. Vol. 2. Biogeography and Systematics. U.S. Dep. Energy, Off. Scient. Techn. Inform., Tennessee.

Garth, J.S. \& T.S. Hopkins. 1968. Pseudocryptochyrus crescentus (Edmondson), a second crab of the corallicolous family Hapalocarcinidae (Crustacea, Decapoda) from the Eastern Pacific with remarks on phragmosis, host specificity, and distribution. Bull. So. Calif. Acad. Sci. 67: 40-48.

Garth, J.S. \& J.W. Stephenson. 1966. Brachyura of the Pacific coast of America. Brachyrhyncha: Portunidae. Allan Hancock Monogr. Mar. Biol. 1: 1-154.

Glassell, S.A. 1936. The Templeton Croker Expedition. I. Six new brachyuran crabs from the Gulf of California. Zoologica 21: 213-218.

Glynn, P.W., N.J. Gassman, C.M. Eakin, J. Cortes, D.B. Smith \& H.M. Guzmán. 1991. Reef coral reproduction in the eastern Pacific: Costa Rica, Panama, and Galapagos islands (Ecuador). I. Pocilloporidae. Mar. Biol. 109: 355-368.

Guinot, D. 1967. Recherches Préliminaires sur les groupements naturels chez les crustacés décapodes brach- youres. II. Les anciens genres Micropanope Stimpson et Medaeus Dana. Bull. Mus. Natn. Hist. Nat. Paris 2: 345-374.

Guinot, D. 1978. Principes d'une classification évolutive des crustacés décapodes brachyoures. Bull. Biol. France Belgique. 112: 211-292.

Guinot, D. 1990. Etablissement du genre Garthiope gen. nov., ses relations avec le genre Coralliope Guinot, 1967, et leurs affinités avec les Trapeziidae sensu lato (Crustacea Decapoda Brachyura). Bull. Mus. Natn. Hist. Nat. Paris 12: 469-487.

Guinot, D. \& T.M. Iliffe. 1991. Garthiope anchialina sp. nov., espèce anchialine des Galapagos, île Isabela, Cueva de la Cadena, avec des remarques sur la faune carcinologique anchialine (Crustacea Decapoda Brachyura). Bull. Mus. Natn. Hist. Nat. Paris 12: 607-621.

Hendrickx, M. 1992. Distribution and zoogeographic affinities of decapod crustaceans of the Gulf of California, Mexico. Proc. San Diego Soc. Nat. Hist. 20: 1-12.

Hendrickx, M. 1993. Crustáceos decápodos del Pacífico Mexicano. p. 271-318. In S.I. Salazar-Vallejo \& N.E. González (eds.). Biodiversidad Marina y Costera de México. CONABIO/CIQRO, México.

Kropp, R.K. 1990. Revision of the genera of gall crabs (Crustacea: Cryptochiridae) ocurring in the Pacific Ocean. Pac. Sci. 44: 417-448.

Markham, J.C., F.E. Donath-Hernández, J.L. VillalobosHiriart \& A. Cantú. 1990. Notes on the shallowwater marine Crustacea of the caribbean coast of Quintana Roo, Mexico. An. Inst. Biol. UNAM 61: 405-446.

Prahl, H. von., F. Guhl \& M. Grögl. 1978. Crustáceos decápodos comensales del coral Pocillopora damicornis L. en la Isla de Gorgona, Colombia. An. Inst. Inv. Mar. Punta Betín 10: 81-93.

Rathbun, M.J. 1918. The grapsoid crabs of America. Bull. U.S. Natl. Mus. 97: 1-461.

Rathbun, M.J. 1930. The cancroid crabs of America of the families Euryalidae, Portunidae, Atelecyclidae, Cancridae and Xanthidae. Bull. U.S. Natl. Mus. 152: 1-593.

Rathbun, M.J. 1937. The oxystomatous an allied crabs of America. Bull. U.S. Natl. Mus. 166: 1-278.

Reyes-Bonilla, H. 1990. Distribución, riqueza específica, aspectos biogeográficos y taxonómicos de los corales hermatípicos del Golfo de California. Tesis de Li- 
cenciatura, Universidad Autónoma de Baja California Sur, La Paz, México.

Reyes-Bonilla, H. 1992. New records for hermatypic corals (Anthozoa: Scleractinia) in the Gulf of California, Mexico, with an historical and biogeographical discussion. J. Nat. Hist. 26: 1163-1175.

Reyes-Bonilla, H. 1993. Biogeografía y ecología de los corales hematípicos (Anthozoa: Scleractinia) del Pacífico de México, p. 207-222. In S.I. Salazar-Vallejo \& N.E. González (eds.). Biodiversidad marina y Costera de México. Comisión Nacional de Biodiversidad/Centro de Investigaciones de Quintana Roo, Ciudad de México.

Robinson, J.A. \& D.A. Thomson. 1992. Status of the Pulmo coral reefs in the lower Gulf of California. Environ. Conserv. 19: 261-264.
Rodríguez de la Cruz, M.C. 1987. Crustáceos decápodos del golfo de California. Secret. Pesca, México. 306 p.

Steinbeck, J. \& E.F. Ricketts. 1941. Sea of Cortez. Viking, Nueva York. 598 p.

Villalobos-Hiriart, J.L., J.C. Nates, A. Cantú, M.D. Valle, P. Flores, E. Lira \& P. Schmidtsdorf. 1989. Listados Faunísticos de México, I. Crustáceos estomatópodos y decápodos intermareales de las islas del golfo de California, México. Inst. Biol. UNAM, México. 114 p. 